\title{
Using systems biology to evaluate targets and mechanism of action of drugs for diabetes comorbidities
}

\author{
Bernd Mayer ${ }^{1}$ \\ Received: 18 November 2015 / Accepted: 18 May 2016/Published online: 4 July 2016 \\ (C) Springer-Verlag Berlin Heidelberg 2016
}

\begin{abstract}
Medications approved for diabetes-associated renal and cardiovascular morbidities and candidate drugs currently in development are subject to substantial variability in drug response. Heterogeneity on a molecular phenotype level is not apparent at clinical presentation, which means that interindividual differences in drug effect at the molecular level are masked. These findings identify the need for optimising patient phenotyping via use of molecular biomarkers for a personalised therapy approach. Molecular diversity may, on the one hand, result from the effect of genetic polymorphisms on drug transport, metabolism and effective target modulation. Equally relevant, differences may be due to molecular pathologies. The presence of distinct molecular phenotypes is suggested by classifiers aimed at modelling progressive disease. Such functions for prognosis incorporate a complex set of clinical variables or a multitude of molecular markers reflecting a diverse set of molecular disease mechanisms. This information on disease pathology and the mechanism of action of the drug needs to be systematically integrated with data on molecular biomarkers to develop an experimental tool for personalising medicine. The large amount of molecular data available for characterising diabetes-associated morbidities allows for elucidation of molecular process model representations of disease pathologies. Selecting biomarker candidates on such grounds and, in turn identifying their association with progressive disease allows for the identification of molecular processes associated with disease progression. The molecular effect of a drug can also be
\end{abstract}

Bernd Mayer

bernd.mayer@emergentec.com

1 emergentec biodevelopment GmbH, Gersthofer Strasse 29-31, 1180 Vienna, Austria modelled at a molecular process level, and the integration of disease pathology and drug effect molecular models reveals candidate biomarkers for assessing drug response. Such tools serve as enrichment strategies aimed at adding precision to drug development and use.

Keywords Biomarker · Diabetic nephropathy · Molecular model $\cdot$ Network $\cdot$ Pharmacogenomics $\cdot$ Prediction $\cdot$ Review

\section{Introduction}

Renal function decline and cardiovascular disease are major morbidities in diabetes mellitus. It has been demonstrated that intensified metabolic and blood pressure control (the latter achieved by the use of agents that block components of the renin-angiotensin-aldosterone system) are effective in decreasing the incidence and slowing the progression of diabetic kidney disease. Nonetheless, many patients still develop nephropathy, whilst there is also a concomitant increase in cardiovascular morbidity and mortality. This finding is evident both in controlled clinical trial settings as well as in everyday clinical practice, in that a substantial fraction of patients with end-stage renal disease have a history of diabetes.

Thus, there is a demand for the development of novel drugs for the treatment of renal and cardiovascular comorbidities. A number of recent clinical trials in this area did not meet their intended endpoints, or had to be stopped early because of adverse events, specifically associated with cardiovascular pathologies [1]. However, some agents currently under clinical investigation are producing promising results, including the sodium/glucose cotransporter 2 (SGLT2) inhibitors [2]. 
Thus, does nephropathy and associated cardiovascular disease in diabetes mellitus occur as a result of a homogeneous clinical and molecular phenotype, or is the clinical presentation afflicted with molecular phenotype heterogeneity? If there is molecular diversity, this may well influence characteristics of disease progression and drug response.

In clinical trials on diabetic kidney disease, the inclusion criteria may be based on established clinical variables commonly used for diagnosis and assessing progression of the disease, e.g. baseline urine albumin/creatinine ratio and estimated glomerular filtration rate, together with levels of $\mathrm{HbA}_{1 \mathrm{c}}$, blood pressure and medication status. The effect of the drug after trial onset often leads to inter-individual variation in these measures. Hence, even when a drug is tested on a cohort of patients who meet a particular set of inclusion criteria, a varied response to the drug is seen. For example, a recent study demonstrated that individual differences may occur in terms of clinical variables and their interactions, and these may be used to predict long-term effects on clinical outcomes, such as doubling of serum creatinine or death [3].

A drug aimed at a molecular target impacts molecular processes and, therefore, if there are different responses to the drug this may imply there is variance in its ability to effectively target a specific molecular process. Additionally, the relevance of the molecular process being targeted may differ depending on the patient's pathology. Following this line of argument, variables that provide information on an individual's molecular phenotype appear to be helpful in the context of selecting a drug based on its mechanism of action. Molecular biomarkers serve as a tool for monitoring the phenotypic status of an individual, whilst also indicating the potential efficacy of a drug with regards to its mechanism of action. Thus, the addition of biomarker assessment to clinical trials promises to provide an insight into drug response variability and could help to identify those individuals who would benefit most from the drug.

\section{Conceptual considerations}

Biomarkers by definition serve as a proxy for the status of a molecular process. Thus, if a molecular biomarker is identified for a clinical context (e.g. a specific pathology) of interest, it then serves as a clinical biomarker. Similarly, if such a biomarker can be assigned to a specific molecular process, this may reflect the relevance of the assigned molecular process with respect to the clinical presentation in focus. For example, if a biomarker is found that identifies patients with accelerated renal function decline, then levels of the biomarker could be evaluated in addition to the established clinical variables characterising the disease, and if it significantly increases prognostic accuracy then the biomarker may become clinically relevant and therefore implemented as a routine part of the test. Interestingly, studies assessing the progression of nephropathy in fairly homogeneous patient cohorts with respect to clinical variables demonstrate the demand for biomarker panels for prognostic accuracy [4].

In renal disease, the functional backgrounds of the individual markers included in such panels identify a number of different molecular processes and pathways, covering a diverse spectrum of molecular mechanisms such as inflammation, stress response, fibrosis and extracellular matrix alterations. These findings support the notion that in diabetic kidney disease a heterogeneous molecular phenotype is determined by the complex interplay of molecular mechanisms in patients who are fairly homogeneous in terms of clinical presentation.

This perspective has significant implications for drug response. Again in general terms, a drug that modulates the function of a specific target first affects the direct molecular process that the target plays a role in. Subsequently, the drug effect triggers downstream molecular processes that are functionally linked to the molecular drug target. Furthermore, this comprehensive drug mechanism of action may affect the molecular background of a specific (personal) pathology. Thus, any variation in patient molecular background may have implications for the drug response and may eventually be reflected at an individual level, ultimately affecting clinical outcomes.

The molecular process interactions that characterise a particular clinical presentation are not only important for studying the effect of a drug, but also for identifying drug target candidates. With biomarkers serving as a proxy for molecular process status we now have the tools for molecular process phenotyping in relation to patient strata-specific disease progression. Furthermore, markers predicting drug response result from integrating the molecular process sets for disease with drug mechanism of action. Including such molecular tools in drug target selection during translational and preclinical research, and subsequently also in clinical trial inclusion criteria for a drug in focus, may well serve to optimise drug selection for specific patient cohorts.

\section{Implementation in diabetic kidney disease}

A scientific literature review identifying molecular mechanisms that are relevant in diabetic kidney disease provides about 30 individual pathways, including the reninangiotensin-aldosterone system, the TGF $\beta$ pathway and extracellular matrix receptor interaction, together with major signalling pathways such as the phosphatidylinositol-4, 5-bisphosphate 3-kinase-AKT (PI3K-AKT), the Janus kinase 
(JAK) - signal transducer and activator of transcription (STAT) system, and chemokine/cytokine signalling.

An alternative strategy for identifying molecular processes integrates molecular signatures from consolidated 'omics' profiling and literature mining to pinpoint molecular features associated with the disease. Using a human protein interaction network allows the feature set to be put into a molecular functional context, i.e. deriving a disease-specific interaction network. Dividing such a network according to topological specifics (e.g. tightly connected gene clusters) provides a molecular process model representation [5]. Intricate molecular interaction within and across various molecular mechanisms becomes evident on the molecular pathway as well as at the molecular process level. Such modelling demonstrates the molecular background of diabetic kidney disease as being a complex regulatory network and the screening of existing scientific literature for drug targets and biomarker candidates reveals a large proportion of the molecular pathways involved. Overall, for diabetic kidney disease, knowledge on molecular processes, drug targets, drug mechanism of action and biomarker candidates provides a comprehensive dataset for systematically linking molecular processes with drug mechanism of action via molecular biomarkers.

The first item to address is the identification of the molecular processes linked to progression of diabetic kidney disease. Here, a molecular process model is used for targeted biomarker candidate selection by, for example, picking a representative candidate for each individual process included in the model. Panel members that show an association with disease progression in experimental testing, in turn, provide information on respective molecular processes involved in progression. It is important to note that adjustment of candidate biomarkers for established clinical variables for assessing progression is counterproductive for the approach discussed here. In contrast to clinical variables, which eventually resemble a surrogate of multiple molecular processes, selected molecular biomarkers are directly associated with individual molecular processes. Also, biomarkers that are collinear with an established clinical variable (in a combined statistical classifier that does not enhance performance to a greater extent than the clinical variable) are of particular value in terms of providing information on relevant molecular processes. Using a disease molecular process model as a basis for biomarker candidate selection for diabetic kidney disease includes matrixmetalloproteases (e.g. MMP2, MMP7), the tyrosine-protein kinase receptor (TEK), and the tumour necrosis factor receptor superfamily, member 1A (TNFRSF1A), amongst others. Experimental testing has shown that such a marker panel adds to prognosis prediction alongside clinical variables, but it is also independently associated with renal function decline [5]. Nonetheless, analysing the biomarker panel readout on an individual patient level identifies variance, implying that certain molecular phenotypes in patients can be defined on the basis of exhibiting a comparable molecular biomarker readout. Thus, the use of biomarkers as molecular process proxies can be used to gain knowledge on patient strataspecific molecular processes resembling a specific path of progressive disease. We can now also use this knowledge for studying the molecular effect of drugs for an identified cohort.

Drug mechanism of action needs to be assessed on at least two distinct molecular levels; the first of which involves pharmacogenomics. An effective drug concentration at the target site is determined by drug transport and metabolism, which may be influenced by genetic polymorphisms in transporters and enzymes [6]. Another genetic element comes in the form of drug target polymorphisms that eventually alter the binding and effective modulation of the target. These effects can be analysed experimentally, e.g. via targeted sequencing. The second molecular level involves the way in which the mechanism of action of a particular drug alters

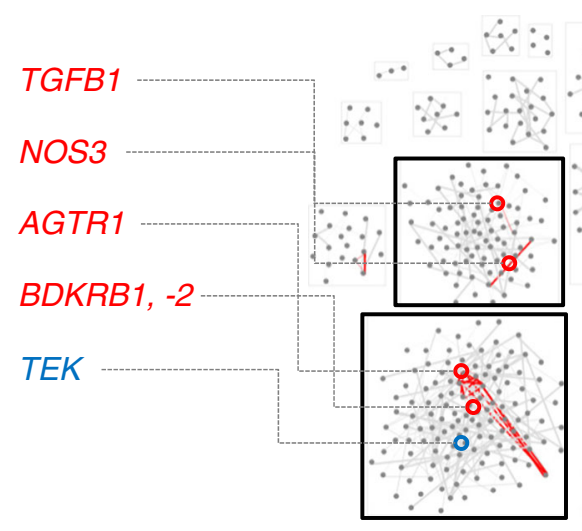

Fig. 1 A molecular process model of diabetic kidney disease and angiotensin receptor blocker mechanism of action interference. The molecular process set (clusters, box borders) holds protein coding genes as nodes (grey) and protein interactions as grey connecting lines. Drug

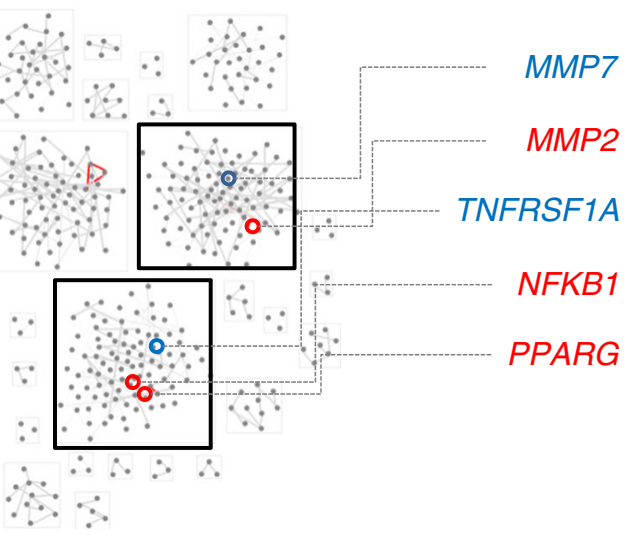

mechanism of action interference is depicted as red nodes and red connecting lines. Four selected molecular processes hold biomarker candidate annotation (blue gene symbols) and specific aspects of drug mechanism of action interference (red gene symbols) 
downstream molecular processes associated with a given molecular pathology. Unfortunately, for the majority of drug classes, information on the drug mechanism of action beyond the direct effect on the target is sparse. Systematic and comprehensive molecular profiling is mainly used for studying drug toxicity aspects. Nevertheless, experimental designs including in vitro models or tissue sample analysis from in vivo studies support drug effect profiling, ideally using transcriptomics or tissue proteomics for obtaining an overall picture of the molecular features being affected by the drug.

For well-established drugs, a set of molecular features associated with the drug effect can be determined by scientific literature mining, as demonstrated for angiotensin receptor blockers [5]. Screening the literature for molecular features affected by, for example, irbesartan and losartan, identifies 125 protein-coding genes. In line with the procedure for deriving a disease pathology molecular process model, a drug mechanism of action molecular model needs to be derived, representing the molecular mechanisms affected by the drug. Having both the drug mechanism of action and disease molecular background in the same model allows for algorithmic interference analysis for the identification of shared molecular process signatures. In this way, a match of drug effect and disease pathology can be approximated on a molecular mechanistic level. In the case of angiotensin receptor blockers and diabetic kidney disease, the shared molecular factors include the direct drug target angiotensin II receptor, type 1 (AGTR1), the bradykinin system (BDKRB1 and 2) further linked with endothelial nitric oxide synthase (NOS3), as well as the NFkB p105 subunit (NFKB1) and the peroxisome proliferator-activated receptor $\gamma$ (PPARG).

Interference analysis provides a drug effect signature for a specific pathology (Fig. 1). This molecular model represents the integration of multiple layers of information: the molecular process map of the pathology of the disease, biomarkers that indicate disease progression, representing the pathophysiology of a subgroup of patients, and an approximation of how the mechanism of action of the drug interferes with such processes. Biomarkers are at the core of experimental testing, first acting as prognostic markers to identify patients with a specific disease progression path, and second to assess the level of interference of the pathological process by the drug.

\section{Perspectives}

Modelling disease pathology and drug mechanism of action on a molecular pathway and process level, and studying biomarker candidates using molecular model interference is a generic procedure broadly expandable to pathologies and drug classes. A key element of this approach is the elucidation of solid molecular biomarker panel data on disease progression together with assignment to molecular processes, be it from rational selection on the basis of process model coverage combined with subsequent experimental testing, or molecular process assignment of biomarkers e.g. derived from explorative profiling approaches. Clinically, such biomarkers support identification of specific molecular phenotypes of progressive disease to expand our understanding of clinical variable variance in relation to outcome. In a translational manner, molecular process information allows for the selection of drug targets embedded in relevant molecular processes, as well as identifying potential interference of molecular mechanisms associated with drug action.

Acknowledgements Andreas Heinzel and Paul Perco (both affiliated with emergentec biodevelopment GmbH, Vienna, Austria) contributed to modelling of diabetic kidney disease pathology and drug mechanism of action.

Funding The work leading to this article received funding from the European Community's Seventh Framework Programme under grant agreement no. 241544 (SysKID) and grant agreement no. 278249 (EU-MASCARA).

Duality of interest The author is Managing Partner of emergentec biodevelopment $\mathrm{GmbH}$, a company developing strategies and computational solutions for biomarker, drug target and drug positioning.

Contribution statement The author is the sole contributor to this paper.

\section{References}

1. Chan GC, Tang SC (2016) Diabetic nephropathy: landmark clinical trials and tribulations. Nephrol Dial Transplant 31:359-368

2. Zinman B, Wanner C, Lachin JM et al (2015) Empagliflozin, cardiovascular outcomes, and mortality in type 2 diabetes. N Engl J Med 373:2117-2128

3. Schievink B, de Zeeuw D, Smink PA et al (2016) Prediction of the effect of atrasentan on renal and heart failure outcomes based on short-term changes in multiple risk markers. Eur J Prev Cardiol 23: 758-768

4. Pena MJ, Heinzel A, Heinze G et al (2015) A panel of novel biomarkers representing different disease pathways improves prediction of renal function decline in type 2 diabetes. PLoS One 10:e120995

5. Lambers Heerspink HJ, Oberbauer R, Perco P et al (2015) Drugs meeting the molecular basis of diabetic kidney disease: bridging from molecular mechanism to personalized medicine. Nephrol Dial Transplant 30:105-112

6. Cavallari LH, Mason DL (2016) Cardiovascular pharmacogenomics - implications for patients with CKD. Adv Chronic Kidney Dis 32: $82-90$ 